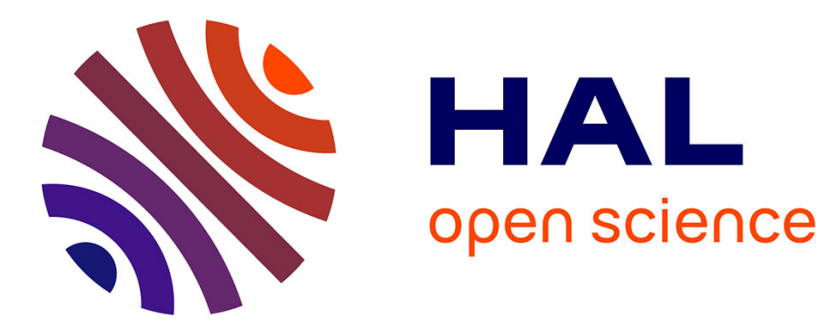

\title{
Pathway Analysis of NAD+ metabolism
}

Luis F de Figueiredo, Toni I Gossmann, Mathias Ziegler, Stefan Schuster

\section{To cite this version:}

Luis F de Figueiredo, Toni I Gossmann, Mathias Ziegler, Stefan Schuster. Pathway Analysis of NAD+ metabolism. Biochemical Journal, 2011, 439 (2), pp.341-348. 10.1042/BJ20110320 . hal-00628678

\section{HAL Id: hal-00628678 \\ https://hal.science/hal-00628678}

Submitted on 4 Oct 2011

HAL is a multi-disciplinary open access archive for the deposit and dissemination of scientific research documents, whether they are published or not. The documents may come from teaching and research institutions in France or abroad, or from public or private research centers.
L'archive ouverte pluridisciplinaire HAL, est destinée au dépôt et à la diffusion de documents scientifiques de niveau recherche, publiés ou non, émanant des établissements d'enseignement et de recherche français ou étrangers, des laboratoires publics ou privés. 


\section{Pathway Analysis of NAD ${ }^{+}$metabolism}

Luis F. de Figueiredo ${ }^{*}, \dagger, \downarrow$, Toni I. Gossmann ${ }^{\S, \|}$, Mathias Ziegler", Stefan Schuster ${ }^{*}$

*Department of Bioinformatics, Friedrich Schiller University Jena, Ernst-Abbe-P1. 2, 07743 Jena, Germany

${ }^{\dagger} \mathrm{PhD}$ Program in Computational Biology, Instituto Gulbenkian de Ciência, 2780-156 Oeiras, Portugal

${ }^{\ddagger}$ Current address: Chemoinformatics and Metabolism, European Bioinformatics Institute (EBI), Wellcome Trust Genome Campus, CB10 1SD, Hinxton, UK

${ }^{\S}$ University of Sussex, School of Life Sciences, BN1 9QG, Brighton, U.K.

"University of Bergen, Department of Molecular Biology, Thormøhlensgate 55, 5008 Bergen, Norway email: Luis.deFigueiredo@ebi.ac.uk, toni.gossmann@googlemail.com,

Mathias.Ziegler@mbi.uib.no,stefan.schu@uni-jena.de

Synopsis

Nicotinamide adenine dinucleotide $\left(\mathrm{NAD}^{+}\right)$is well known as a crucial cofactor in the redox balance of metabolism. Moreover, $\mathrm{NAD}^{+}$is degraded in ADP-ribosyl transfer reactions which are important components of multitudinous signalling reactions. These include reactions linked to DNA repair and ageing. Here, using the concept of elementary flux modes (EFMs), we established all potential routes in a network describing $\mathrm{NAD}^{+}$biosynthesis and degradation. All known biosynthetic pathways, which include de novo synthesis starting from tryptophan as well as the classical Preiss-Handler pathway and $\mathrm{NAD}^{+}$synthesis from other vitamin precursors, were detected as EFMs. Moreover, several EFMs were found that degrade $\mathrm{NAD}^{+}$, represent futile cycles or have other functionalities. The systematic analysis and comparison of the networks specific for yeast and humans documents significant differences between species with regard to both the use of precursors, biosynthetic routes and $\mathrm{NAD}^{+}$dependent signalling.

Key words

$\mathrm{NAD}^{+}$synthesis, $\mathrm{NAD}^{+}$salvage pathways, niacin, elementary flux modes, Preiss-Handler pathway, Sirtuins

Abbreviations used: Bna6, quinolinate phosphoribosyl transferase; Isn1, IMP-specific 5'nucleotidase 1; NA, nicotinic acid; NaAD, deamino-NAD ${ }^{+}$; Nam, nicotinamide; NaMN, nicotinic acid mononucleotide; NAMPT, nicotinamide phosphoribosyl transferase; NAR, nicotinate D-ribonucleoside; Nma1,2, nicotinamide/nicotinate-nucleotide adenylyltransferase 1,2; NMN, nicotinamide mononucleotide; Npt1, nicotinate phosphoribosyl transferase; Npy1, $\mathrm{NAD}^{+}$pyrophosphatase; NR, N-ribosylnicotinamide; Nrk1, nicotinamide riboside kinase; Pnc1, nicotinamidase 1; Pnp1, purine nucleoside phosphorylase 1; PRPP, 5-phospho- $\alpha$-Dribose-1-diphosphate; Prs1-5, ribose-phosphate pyrophosphokinases 1-5; QA, quinolinic acid; Qns1, glutamine-dependent NAD ${ }^{+}$synthase; ribose-P, ribose-phosphate; Sdt1, pyrimidine 5'nucleotidase 1; Urh1, uridine hydrolase 1. 


\section{Introduction}

Nicotinamide adenine dinucleotide $\left(\mathrm{NAD}^{+}\right)$is well known as a co-enzyme of pivotal importance in the redox balance of metabolism, as it is continuously interconverted between an oxidized $\left(\mathrm{NAD}^{+}\right)$and reduced (NADH) state. In the terminology of network theory [1], it is a "hub" because of its high connectivity, that is, the large number of reactions in which it participates. The reaction database in KEGG (release 51.0, [2]) identifies around 1600 reactions that use pyridine nucleotides for hydride transfer (all organisms stored in KEGG taken together).

Consumption of $\mathrm{NAD}^{+}$involves release of nicotinamide (Nam) and transfer of the remaining ADP-ribose moiety onto acceptor molecules. ADP-ribosyl transfer reactions are key constituents of intracellular signalling networks that control vital processes [3]. Protein acceptors are modified by mono-ADP-ribosylation, poly-ADP-ribosylation or $\mathrm{NAD}^{+}$dependent deacetylation and thereby undergo changes of their functional properties [3]. PolyADP-ribosylation appears to be confined to the nucleus of multicellular organisms and is involved in a variety of processes involving DNA rearrangements, transcription and repair [4]. $\mathrm{NAD}^{+}$-dependent deacetylases (EC 3.5.1.-) represent a conserved class of enzymes [5] that transfer the acetyl group from modified proteins onto the ADP-ribose moiety liberated from $\mathrm{NAD}^{+}$. They are also referred to as Sirtuins (based on the founding member, yeast Sir2), which also include structurally related mono-ADP-ribosyltransferases [5]. Sirtuins have a critical impact on the regulation of nuclear events (e.g., by histone deacetylation), but also on central metabolic enzymes such as acetyl-CoA synthase, carbamoyl phosphate synthase and glutamate dehydrogenase $[5,6]$. Sirtuins have been found in prokaryotes and eukaryotes including yeast and mammals. However, neither mono- nor poly-ADP-ribosylation have been detected in yeast. Moreover, $\mathrm{NAD}^{+}$glycohydrolase (NADase) activity is also absent from yeast. NADases (EC 3.2.2.5) cleave $\mathrm{NAD}^{+}$into ADP-ribose (that is, ADP-ribosyl transfer onto water) or generate cyclic ADP-ribose by forming an intramolecular bond between the terminal ribose and the adenine ring [7]. Both ADP-ribose and cyclic ADP-ribose are potent intracellular calcium messengers acting on TRPM2 channels [8] or ryanodine receptors [9], respectively. Given the wide spectrum and permanent activity of $\mathrm{NAD}^{+}$consuming reactions, it is obvious that cellular $\mathrm{NAD}^{+}$pools need to be continuously replenished [10].

A well known route of NAD ${ }^{+}$synthesis is the Preiss-Handler pathway $[11,12]$. In that pathway, nicotinic acid (NA) is converted to $\mathrm{NAD}^{+}$by consecutive addition of ribosephosphate (from phosphoribosyl pyrophosphate, PRPP), AMP (from ATP) and an amino group (form ammonium or glutamine). Another widely found biosynthetic pathway of $\mathrm{NAD}^{+}$, often referred to as de novo synthesis, starts from tryptophan (cf. [10, 13]). A number of lower organisms can alternatively synthesize $\mathrm{NAD}^{+}$de novo from aspartate [13]. These routes, too, require amidation of the NA moiety to $\mathrm{NAD}^{+}$. Nam is also used as $\mathrm{NAD}^{+}$precursor. Interestingly, some, primarily lower, organisms deamidate Nam to NA, which is then used in the Preiss-Handler pathway, whereas other organisms convert Nam more directly to NAD ${ }^{+}$ via nicotinamide mononucleotide (NMN) formation (cf. Fig. 1). NA and Nam are collectively known as niacin or vitamin $\mathrm{B}_{3}$ and represent physiological precursors of $\mathrm{NAD}^{+}$in mammals (cf. [14]). Lack of niacin in the diet leads to the disease of pellagra. Tryptophan appears to be insufficient to fully maintain mammalian $\mathrm{NAD}^{+}$levels [14], but it may have an important role as NAD ${ }^{+}$precursor in some tissues (cf. [15]). Recent work identified the ribosides of NA and Nam (NAR and NR, respectively) as additional precursors which enter $\mathrm{NAD}^{+}$synthesis by alternative routes [16]. Whether riboside precursors can fully complement mammalian niacin deficiency is not known.

To understand and explore the emerging complexity of $\mathrm{NAD}^{+}$metabolism, a systematic analysis of the network comprising its biosynthesis and catabolic conversions is required. This can be performed by elementary-modes analysis [17]. Many metabolites can be synthesized or degraded on multiple routes, possibly with different molar yields. Elementary 
modes provide a powerful tool for determining the degree of multiplicity and, thus, of redundancy [18] and to identify routes with maximum yields or minimal energy expenditure $[17,19]$. An elementary flux mode (EFM) is a minimal set of enzymes that is stoichiometrically and thermodynamically feasible at steady state [17]. Any flux distribution in the living cell is a superposition of EFMs [20]. EFM analysis has been successfully applied to various systems such as central metabolism in Escherichia coli [18] and fatty acid synthesis in plants [21]; for review see [19]. Moreover, EFMs can readily be used to assess the impact of nutrients. This is done by checking which modes start from given nutrients and, thus, would be lost in their absence [22], and which conversions are infeasible even in their presence because the routes are stoichiometrically not balanced [23].

In this study, we first focus on a theoretical analysis of $\mathrm{NAD}^{+}$biosynthetic pathways in a generic network. We then compare the network characteristics of NAD synthesis in mammals and yeast. As pointed out above, in yeasts, the variety of $\mathrm{NAD}^{+}$-degrading signalling reactions appears to be limited to a single type (NAD ${ }^{+}$-dependent deacetylation), whereas in mammals, it is far more extensive with regard to both the number of reaction types and processes they control. Therefore, comparative analysis of these two clades may provide information about the evolution of signal transduction. The analyses presented here provide a basis for the experimental verification of the role of identified EFMs and the development of a kinetic model of $\mathrm{NAD}^{+}$biosynthesis.

\section{Methods}

\subsection{Metabolic model and data sources}

The reaction scheme analysed here is depicted in Fig. 1 and is described in more detail in the Supplementary material. It represents a generic model covering the situation in many organisms, notably mammals and many yeasts such as Saccharomyces cerevisiae, Schizosaccharomyces pombe, and Candida albicans (cf. [13]). Organism-specific routes will be discussed below. The directionality of reactions was defined according to the binary distinction made in the original literature and the database BRENDA [24]. Information about the directionality is in many cases scarce. When necessary, we complemented that information based on established knowledge about similar reactions in other biochemical pathways.

In EFM analysis (and also in other modelling analyses of metabolism), a distinction is made between internal and external metabolites [17, 20]. Internal substances are assumed to be at steady state by a balance between production and consumption. External metabolites are assumed to have constant concentrations, because they are available in large excess or are exchanged across the boundaries of the system. The network (Fig. 1) contains the input of quinolinic acid (QA) generated from tryptophan and five exchange reactions associated with the metabolic pools of nicotinamide (Nam), nicotinic acid (NA), nicotinate D-ribonucleoside (NAR), N-ribosylnicotinamide (NR) and $\mathrm{NAD}^{+}$. Very little is known about transport reactions associated with these $\mathrm{NAD}^{+}$precursors and intermediates and about the exact localization of the enzymes in the network. Therefore, we do not consider compartmentation. Moreover, the exchange reactions are to be interpreted in a more general sense. The corresponding substances might be degraded, converted into other substances or built from other compounds. Nevertheless, it is known that at least some of these substances can enter the cell, for example Nam and NA (vitamin $\mathrm{B}_{3}$ ) or NR coming from the diet [16]. We denoted the exchange reactions by the suffix "_pool" and the metabolites present in these pools by the suffix “_ex”.

An additional set of metabolites, such as ATP, ribose, ribose phosphate (ribose-P; corresponding both to ribose 5-phosphate and ribose 1-phosphate), glutamine, and glutamate were also considered external. This simplification was made in order to reduce the complexity of the problem and thereby focus only on the balance of the pyridine ring and 5-phospho- $\alpha-\mathrm{D}-$ 
ribose-1-diphosphate (PRPP). We included the reaction catalysed by ribose-phosphate pyrophosphokinases 1-5 (Prs1-5) so that the overall reaction associated to each EFM can be represented correctly in terms of ATP, ribose or ribose-P requirements. Since PRPP is an energy-rich compound, fixing it as external would lead to underestimation of the ATP demand. Note that the inclusion of Prs1-5 does not change the number nor the structure of the elementary flux modes computed from the network.

The presence of the corresponding genes in yeast and various human cells such as hepatocytes was verified based on the databases KEGG [2], BioCyc [25] and the literature (see Supplementary material for more details).

\section{[Figure 1 here]}

The presence of nicotinamidase (Pnc1, EC 3.5.1.19), in many bacteria and fungi (including yeasts) has been known for a long time. Recently, homologues of nicotinamidase were identified in Caenorhabditis elegans [26] and in Drosophila melanogaster [27]. NAD ${ }^{+}$ synthase (Qns1, EC 6.3.5.1), in humans operates by using glutamine [28]. The consumption (labelled ADP-ribosyl transfer reaction in Fig. 1) reaction corresponds to a set of transformations all starting with the release of the ADP-ribose moiety. This set includes the mere hydrolysis, hydrolysis and subsequent conversion into cyclic ADP ribose by $\mathrm{NAD}^{+}$ glycohydrolases (EC 3.2.2.5), hydrolysis and subsequent polymerisation to poly-ADP ribose by poly(ADP-ribose) polymerase (EC 2.4.2.30) and the transfer of ADP ribose to other moieties, such as arginine or acetyl (e.g. by SIRTUINs, see Introduction). We use the term ADP-ribosyl transfer as a general term even if ADP-ribose is just released or transformed into cyclic or poly-ADP ribose.

The export reaction Nam_pool involves, among others, the accumulation of Nam and its methylation by nicotinamide N-methyltransferase (EC 2.1.1.1). The enzyme NAMPT is absent from yeast and constitutes the main route of Nam salvage in humans [29]. Recently, yeast uridine hydrolase, Urh1, was found to catalyse the conversion of NAR and NR to NA and Nam, respectively [30, 31].

The reactions catalyzed by Npt1 and NAMPT were set to irreversible in the direction of ribonucleotide synthesis. Indeed, in the presence of ATP, the reaction equilibrium is shifted towards the ribonucleotide formation, because it is coupled with the hydrolysis of ATP [32, 33]. Although the exact ATP stoichiometry is unknown in the case of NAMPT, because the coupling is weak [33], we here use a 1:1 stoichiometry for both reactions of ribonucleotide formation.

\subsection{EFM analysis}

EFMs were computed by the program Metatool 5.1 [34] which is freely available from our website http://pinguin.biologie.uni-jena.de/bioinformatik/networks/. In the network depicted in Fig. 1, several enzymes can catalyze two reactions due to broad substrate specificity. In the model, these reactions were distinguished by adding a suffix ' 2 ' to one of the reactions. In this analysis we considered the presence of a given enzyme when at least one of the associated reactions is present. The EFMs are classified in several subsets according to their presence in the organisms and to their functionality.

A tool for visualizing EFMs was developed using the software GraphMLViewer ( $y$ Works $\mathrm{GmbH}$, Tübingen, Germany). The tool can be used online on our website http://pinguin.biologie.uni-jena.de/bioinformatik/networks/ (Access credential during the review process- username:BiochemicalJ password:kynurenine26). 


\section{Results}

\subsection{Overview of EFMs}

The generic model gave rise to 113 EFMs listed in the Supplementary Material. They can be visualized by the tool mentioned in Section 2.2 (http://pinguin.biologie.unijena.de/bioinformatik/networks/; see above the details to access the tool). Three out of these EFMs contain enzymes absent from humans (Urh1 and Pnc1) and NAMPT not present in yeast. We did not immediately discard them, because they might be relevant in other organisms. In Fig. 2, eight selected EFMs are depicted.

\section{[Figure 2 here]}

In the metabolic network under study, there are 12 EFMs that produce NAD ${ }^{+}$. The de novo synthesis from QA (kynurenine pathway) is represented in EFM 26 (Fig. 2a) and the PreissHandler pathway converting NA to $\mathrm{NAD}^{+}[11,12]$ (including the balancing of PRPP by synthesis from ribose-P) is represented in EFM 25 (Fig. 2b). There are three pathways associated with $\mathrm{NAD}^{+}$synthesis from NAR, all experimentally verified. One of them (mode 22) is Nrk-dependent [35] and two are Nrk-independent [31]. Among the latter, one is yeast specific, using Urh1 (mode 24) and the other is present in both organisms, using Pnp (mode 23). Both involve the Preiss-Handler pathway. There are 7 EFMs that start from amidated precursors, two from Nam and five from NR. One of the pathways for $\mathrm{NAD}^{+}$synthesis from Nam is yeast-specific, using Pnc1 and then the Preiss-Handler pathway (mode 20) [36], while the other is specific for humans. Mode 19 uses NAMPT to convert Nam to NMN, which is then converted to $\mathrm{NAD}^{+}$by NMNAT1-3 (the human homologues of the yeast enzymes Nam1,2) [37, 38]. Starting from NR, there is one EFM specific for humans (mode 29), which is Nrk1-independent and uses NAMPT and PNP (Pnp1 in yeast), and two that are exclusive for yeast (represented by modes 27 and 28, the latter is shown in Fig. 2e). Both take a detour via NA involving the yeast-specific enzyme Pncl and then use the Preiss-Handler pathway [39] and are Nrk1-independent as well. The Nrk-dependent pathway represented by mode 21 is present in both organisms [16].

Net consumption of $\mathrm{NAD}^{+}$leads to the generation of NA, NAR, Nam or NR in yeast and Nam or NR in humans. QA cannot be produced from $\mathrm{NAD}^{+}$due to the irreversibility of the reaction catalyzed by Bna6. There are in total 18 routes with $\mathrm{NAD}^{+}$net consumption, four of which proceed via the ADP-ribosyl transfer reaction and therefore, play a role in signalling.

An interesting observation is the number of EFMs that constitute interconversions between $\mathrm{NAD}^{+}$precursors. These EFMs account for $\sim 2 / 3$ of the EFMs computed from this network. For these interconversions enzymes Qns1 and Nma1,2 play an important role, being present in $\sim 80 \%$ of them (Fig. 3). However, the relevant number is likely to be lower, because of compartmentation - NMNATs 2 and 3 (human homologues of yeast Nma1,2) are located in the Golgi complex and mitochondria, respectively [40]. It is largely unknown which $\mathrm{NAD}^{+}$ derivatives can cross intracellular membranes and how the biosynthetic enzymes are distributed. Permeability constraints may thus reduce the number of these pathways.

\section{[Table 1 here]}

It is of interest to note that several EFMs in the network constitute futile cycles. Generally, futile cycles are defined as routes with no net transformation except hydrolysis of energy-rich compounds. There are five EFMs satisfying this definition. Two of them (modes 41 and 42) represent the overall transformation ATP $\rightarrow \mathrm{P}_{\mathrm{i}}+\mathrm{ADP}$ and consist of the enzymes Nrk1 and Sdt1/Isn1. Mode 40 has the overall stoichiometry ATP $+\mathrm{H}_{2} \mathrm{O} \rightarrow \mathrm{PP}_{\mathrm{i}}+\mathrm{AMP}$ and is composed of the enzymes Nam1,2 and Npy1. Interestingly, only four of these futile cycles are present in yeast, whereas the human model involves one additional futile cycle (mode 47, Fig. 2c) 
composed of the reactions catalyzed by NAMPT, NT5 (the human homologue of the yeast enzymes Sdt1 and Isn1) and PNP. In a less strict sense, cyclic modes with no net consumption of $\mathrm{NAD}^{+}$or its precursors can be considered as futile cycles as well. Applying this definition, we find another six futile cycles in the network (Supplementary Material). Two of them result in the release of the amino group from glutamine. These modes require the enzymes Pnc1 and Qns1. Two of the futile cycles in the broader sense convert ribose-P into ribose and another two perform both this conversion and the deamidation of glutamine. Interestingly, these more broadly defined futile cycles require enzymes specific to yeast and are therefore absent in humans.

The presence of many futile cycles in the network can be explained by the existence of several enzymes catalysing similar reactions with opposite directions. Whether these futile cycles are of physiological relevance depends on compartmentation (not considered here) and on regulation in the cell. In fact, it was recently shown that Sdt1 and Isn1 are highly regulated [41]. For example, it was shown that Isn1 levels decrease when yeast is growing in low glucose medium [41]. Taking into account the fact that Isn1 takes part in many of the futile cycles, the regulatory effect can be seen as a way to reduce energy dissipation and thereby avoiding wasting resources. Therefore, detection of potential futile cycles by EFM analysis may indeed be helpful to identify critical nodes that require regulation (cf. [17]). Further data and studies on enzyme regulation and localization are required to determine the physiological significance of these cycles.

There are 21 EFMs that include ADP-ribosyl transfer (indicated as producing ADPribosyl-X). These EFMs account for $\mathrm{NAD}^{+}$net consumption. Interestingly, the majority of EFMs including generation of ADP-ribosyl-X also involve interconversion of precursors. There are two cyclic EFMs that produce ADP-ribosyl-X without net consumption of precursors. One of them is present only in yeast (mode 51, Fig. 2g) with the overall stoichiometry 4 ATP + gln $+2 \mathrm{H}_{2} \mathrm{O}+$ ribose-P $\rightarrow 3 \mathrm{PP}_{\mathrm{i}}+\mathrm{ADP}+$ glu $+\mathrm{NH}_{3}+2 \mathrm{AMP}+$ ADP-ribosyl-X. The other is specific for humans (mode 52, Fig. 2h) with the overall stoichiometry $3 \mathrm{ATP}+$ ribose-P $\rightarrow 2 \mathrm{PPi}+\mathrm{AMP}+\mathrm{ADP}-$ ribosyl-X (Figs. $2 \mathrm{~g}$, h, respectively). The human pathway (Fig. 2h) is of great physiological significance, because NAMPT is considered to be rate limiting and is subject to circadian control [42, 43].

Considering the energetic requirements of $\mathrm{NAD}^{+}$biosynthetic pathways, it is evident that, on average, about five ATP are required to generate one molecule of $\mathrm{NAD}^{+}$(Table 1). There is one EFM (mode 30) that is not present in any of the two organisms because it involves both Urh and NAMPT, which do not occur together in either organism. The least expensive pathways correspond to the salvage of the amidated $\mathrm{NAD}^{+}$precursors, NR and Nam. For example, mode 21, present in both organisms, starts from NR following the Nrk1-dependent pathway and requires only two ATP. Three pathways require four ATPs. Two modes (modes 29 and 19) which use the amidated precursors NR and Nam, respectively are only present in humans. Mode 22 (Figure 2d) uses NAR as precursor. Four EFMs, exclusively present in yeast (EFMs 20, 24, 27 and 28), require more ATP for $\mathrm{NAD}^{+}$synthesis. One third of the pathways require one mole of ribose-P.

\subsection{Importance of enzymes within pathways}

In Fig. 3, the frequency by which each enzyme is found in an EFM belonging to a specific subset of EFMs is represented. For example, Pnc1, which generates ammonium, is absent in humans and, consequently, there is no futile cycle (in the broader sense) of glutamine deamidation involving Qns1, as opposed to what is observed for yeast. On the other hand, Nma1,2 is present in many EFMs, being essential for $\mathrm{NAD}^{+}$synthesis in both organisms.

It is interesting to see, in Fig. 3, that Sdt1 and Isn1 have a more preponderant role than ADP-ribosyl transfer in the EFMs consuming $\mathrm{NAD}^{+}$. In humans, all the EFMs consuming $\mathrm{NAD}^{+}$convert it to Nam or NR. In general, our analysis indicates that the $\mathrm{NAD}^{+}$metabolism 
of humans is more centered around the amidated ribonucleotides. In contrast, Npt1 plays an important role in the $\mathrm{NAD}^{+}$metabolism of yeast. In yeast, there are few EFMs producing NR from $\mathrm{NAD}^{+}$when comparing with the other three precursors.

In general, there are not many significant differences between the frequencies in which reactions occur in the subsets of both organisms, in spite of the network differences. It seems that the network differences have a bigger impact on the number of EFMs belonging to each subset. For example the subsets of EFMs corresponding to $\mathrm{NAD}^{+}$precursor inter-conversion and $\mathrm{NAD}^{+}$comsumption have more EFMs in yeast than in humans. Moreover, more than two thirds of the EFMs in yeast are specific for this organism.

\section{[Figure 3 here]}

\section{Discussion}

Here, we have performed a topological analysis of $\mathrm{NAD}^{+}$metabolism by using the concept of elementary flux modes (EFMs). This provides a systematic overview of the metabolic capabilities of that system including interesting effects on signalling. There is increasing interest in large-scale and even whole-cell metabolic models [44] and methods for topological analysis of such large networks are becoming available [45, 46]. However, medium-sized models describe in detail specific parts of metabolism and proyide a valuable resource as well. They permit to derive non-trivial conclusions of great biochemical interest, which are easier to interpret in several aspects than the results from a large-scale model. Thus, we here consider a relatively small, yet complex system to study the interconnectivity between $\mathrm{NAD}^{+}$ metabolism and signalling pathways. The network analysed here includes 23 reactions and is complicated enough that the entire set of pathways across the system cannot easily be seen by inspection. Consequently, we used computational methods to deal with the combinatorial complexity of the metabolic network and systematically, comprehensively and rapidly enumerated all the metabolic pathways enclosed in its structure. Moreover, we have included recently identified enzymatic reactions into the network, which are not yet included in wholecell models.

The system under study only involves unit stoichiometric coefficients. Thus, path-finding methods based on graph theory [47] might be used. There has been a debate in the literature about the pros and cons of stoichiometric methods (as used here) and path-finding methods $[23,47,48]$. In our view, application of stoichiometric network methods was straightforward, rather than to check whether path-finding methods would have been sufficient to describe the metabolic system investigated.

We have considered the synthesis and degradation of $\mathrm{NAD}^{+}$. We have not included the hydride transfer between $\mathrm{NAD}^{+}$and $\mathrm{NADH}$, because this is involved in a huge number of redox reactions. Moreover, these reactions are irrelevant for the buildup and degradation of $\mathrm{NAD}^{+}$and the associated signalling processes. The detected EFMs comprise synthetic routes not only for $\mathrm{NAD}^{+}$, but also for relevant $\mathrm{NAD}^{+}$precursors such as N-ribosylnicotinamide (NR). From the 113 EFMs computed for the generic network, 100 correspond to yeast, 50 correspond to humans of which 40 are common to both organisms, and 3 do not occur in any of the two organisms. The higher abundance of pathways in yeast is surprising, because of the more diverse ways of $\mathrm{NAD}^{+}$-related signalling in humans. Moreover, a general tendency is that the human network is primarily based on pathways involving the amidated forms of the $\mathrm{NAD}^{+}$derivatives while yeast uses preferentially the deamidated forms.

Several of the obtained EFMs can be assigned to well-known pathways. The PreissHandler pathway $[11,12]$ produces $\mathrm{NAD}^{+}$from nicotinic acid (NA), which humans must take up in their diet. To what extent intestinal flora produces NA as $\mathrm{NAD}^{+}$precursor remains to be determined [14]. One specific observation is that $\mathrm{Na}$ is produced from $\mathrm{Nam}$ by deamidation by bacterial nicotinamidase in the gut (cf. [14]). 
The kynurenine pathway has also been found in the form of an EFM. It provides quinolinic acid (QA) as $\mathrm{NAD}^{+}$precursor, which originates from tryptophan, Moreover, we have identified the EFMs for the Preiss-Handler independent $\mathrm{NAD}^{+}$synthesis routes present in fungi and humans, which start from NR. They use either Nrk [16] or the more recently described Nrk-independent pathway [39]. NR was identified in cow milk [16]. Thus, it can be speculated that it is used as a precursor for $\mathrm{NAD}^{+}$by infants and animal sucklings, since milk is low in NA [49]. Moreover, it is interesting that the most economical pathways in terms of ATP consumption are those salvaging the amidated $\mathrm{NAD}^{+}$precursors, NR and nicotinamide (Nam). Another interesting nutritional fact is that corn has only very limited contents of NA without being enriched by other $\mathrm{NAD}^{+}$precursors. Thus, a predominant consumption of corn can cause pellagra [49].

Many of the above-mentioned biosynthesis/salvage pathways are likely to occur in the liver, kidney and small intestine (cf. $[15,38,50]$ ). Thus, these organs would be responsible for the levelling of Nam in the serum and, consequently, mainly pathways producing Nam are operative therein, while other organs may preferentially use Nam for $\mathrm{NAD}^{+}$generation. Our analyses indicate that, in yeast, nicotinate D-ribonucleoside (NAR) can be made from NR, Nam, NA and QA, while in humans, it can only be produced from NA and QA. The tissue distribution of the various pathways in humans could thus reveal interesting interdependencies between different organs with regard to $\mathrm{NAD}^{+}$supply.

We found two EFMs that do not involve net formation or use of $\mathrm{NAD}^{+}$(apart from the futile cycles). One of them is present in yeast and the other in humans (Fig. 2g,h). Both transform ribose-phosphate and ATP into an ADP-ribosyl moiety and proceed via Nam. This indicates that $\mathrm{NAD}^{+}$-dependent signalling can proceed without net consumption of $\mathrm{NAD}^{+}$and that $\mathrm{NAD}^{+}$signaling activity can be investigated by measuring the Nam pool. Thus, at steady state, signalling need not involve a loss in the $\mathrm{NAD}^{+} / \mathrm{NADH}$ pool. Whether these pathways are indeed at steady state and physiologically relevant depends on kinetics and regulation, which we have not considered here. It was recently found $[42,43]$ that the $\mathrm{NAD}^{+}$salvage pathway shown in Fig. $2 \mathrm{~h}$ is under control of the circadian clock. Both the expression of NAMPT and the liver $\mathrm{NAD}^{+}$levels oscillate in a clock-dependent manner. These experimental findings highlight the importance of Nam utilization by NAMPRT in mammals, as they point to a strong link between the circadian clock and $\mathrm{NAD}^{+}$metabolism.

Pathway and flux analyses in the $\mathrm{NAD}^{+}$network are of interest in the framework of research related to ageing. Several hypotheses on the role of the positive effect of calorie restriction on longevity and its relation to $\mathrm{NAD}^{+}$metabolism have been put forward (cf. [41, 51]). One of them states that calorie restriction increases $\mathrm{NAD}^{+}$turnover without altering steady-state $\mathrm{NAD}^{+}$levels $[41,52]$. This suggestion would correspond to the cyclic modes consuming ribose phosphate and producing ADP-ribosyl-X. However, it appears questionable whether increased consumption of ribose for $\mathrm{NAD}^{+}$turnover is compatible with calorie restriction.

It is of interest to compare our results with mutation studies. For example, the mutant strain $\Delta n r k 1 \Delta u r h 1 \Delta p n p l$ of $S$. cerevisiae was studied and it was found that it accumulates Nam when NA is supplied in the growth medium [41]. When deleting the enzymes Nrk1, Urh1 and Pnp1, 23 EFMs remain, including a cyclic mode corresponding to steady-state signalling. Five out of these EFMs consume $\mathrm{NAD}^{+}$and three produce $\mathrm{NAD}^{+}$from QA, NA and Nam. From the former, there are two pathways producing NR; the remaining three produce one of the following precursors: NA, Nam and NAR. NR is also an important precursor for $\mathrm{NAD}^{+}$synthesis in other mutant strains. This allows cross-feeding between the wildtype of $S$. cerevisiae, which can secrete NR, and mutants that take it up [53].

We have made a distinction between futile cycles in the strict and in the broader sense. The overall transformation of the former is a hydrolysis of ATP. The latter hydrolyse ribosephosphate or deamidate glutamine. Thus, the definition of futile cycles depends on what is 
considered as a cofactor. Moreover, there are cyclic routes producing ADP-ribosyl-X. These play a role in signalling and, thus, form a bridge between metabolism and regulation.

We have not considered compartmentation in the present model, as this would have significantly increased complexity. Moreover, the subcellular distribution of $\mathrm{NAD}^{+}$ biosynthetic enzymes as well as the presence of relevant metabolite carriers in organellar membranes still need to be conclusively determined. The present EFM analysis in yeast and humans is in agreement with experimental observations and thus provides a valuable tool for similar analyses of bacteria (e.g. enterobacteria, relevant for humans) and other clades. The generic model includes all possible conversions presently known and can thus readily be adopted to the specific subsets in other organisms.

\section{Acknowledgements}

The groups in which L.F.F. and St.S. work belong to the Jena Centre for Bioinformatics (JCB) and Jena School of Microbial Communication (JSMC). The scientific atmosphere in these networks has significantly stimulated this work. The authors acknowledge stimulating discussions with Ines Heiland, Michael Ristow and Anne-Kristin Stavrum and are grateful to Ina Weiss for helpful literature search.

\section{Funding}

Portuguese entities: Fundação Calouste Gulbenkian, Fundação para a Ciência e a Tecnologia (FCT) and Siemens SA Portugal [PhD grant SFRH/BD/32961/2006 to L.F.F.]. German Ministry of Education and Research (BMBF) within the Virtual Liver (grant no. 0315758) and GerontoSys programmes (grant no. 0315581D). 


\section{References}

1 Jeong, H., Tombor, B., Albert, R., Oltvai, Z. N. and Barabasi, A. L. (2000) The largescale organization of metabolic networks. Nature. 407, 651-654

2 Kanehisa, M., Goto, S., Furumichi, M., Tanabe, M. and Hirakawa, M. (2010) KEGG for representation and analysis of molecular networks involving diseases and drugs. Nucleic Acids Res. 38, D355-360

3 Berger, F., Ramirez-Hernandez, M. H. and Ziegler, M. (2004) The new life of a centenarian: signalling functions of NAD(P). Trends Biochem Sci. 29, 111-118

4 Schreiber, V., Dantzer, F., Ame, J. C. and de Murcia, G. (2006) Poly(ADP-ribose): novel functions for an old molecule. Nat Rev Mol Cell Biol. 7, 517-528

5 Imai, S. and Guarente, L. Ten years of NAD-dependent SIR2 family deacetylases: implications for metabolic diseases. Trends Pharmacol Sci. 31, 212-220

6 Huang, J. Y., Hirschey, M. D., Shimazu, T., Ho, L. and Verdin, E. Mitochondrial sirtuins. Biochim Biophys Acta. 1804, 1645-1651

7 Lee, H. C. (2000) Enzymatic functions and structures of CD38 and homologs. Chem Immunol. 75, 39-59

8 Naziroglu, M. (2007) New molecular mechanisms on the activation of TRPM2 channels by oxidative stress and ADP-ribose. Neurochem Res. 32, 1990-2001

9 Galione, A. (1994) Cyclic ADP-ribose, the ADP-ribosyl cyclase pathway and calcium signalling. Mol Cell Endocrinol. 98, 125-131

10 Magni, G., Amici, A., Emanuelli, M., Orsomando, G., Raffaelli, N. and Ruggieri, S.

(2004) Enzymology of NAD ${ }^{+}$homeostasis in man. Cell Mol Life Sci. 61, 19-34

11 Preiss, J. and Handler, P. (1958) Biosynthesis of diphosphopyridine nucleotide. I. Identification of intermediates. J Biol Chem. 233, 488-492

12 Preiss, J. and Handler, P. (1958) Biosynthesis of diphosphopyridine nucleotide. II. Enzymatic aspects. J Biol Chem. 233, 493-500

13 Gazzaniga, F., Stebbins, R., Chang, S. Z., McPeek, M. A. and Brenner, C. (2009) Microbial NAD metabolism: lessons from comparative genomics. Microbiol Mol Biol Rev. 73, 529-541

14 Bogan, K. L. and Brenner, C. (2008) Nicotinic acid, nicotinamide, and nicotinamide riboside: a molecular evaluation of $\mathrm{NAD}^{+}$precursor vitamins in human nutrition. Annu Rev Nutr. 28, 115-130

15 Bernofsky, C. (1980) Physiology aspects of pyridine nucleotide regulation in mammals. Mol Cell Biochem. 33, 135-143

16 Bieganowski, P. and Brenner, C. (2004) Discoveries of nicotinamide riboside as a nutrient and conserved NRK genes establish a Preiss-Handler independent route to $\mathrm{NAD}^{+}$in fungi and humans. Cell. 117, 495-502

17 Schuster, S., Fell, D. A. and Dandekar, T. (2000) A general definition of metabolic pathways useful for systematic organization and analysis of complex metabolic networks. Nat Biotechnol. 18, 326-332

18 Stelling, J., Klamt, S., Bettenbrock, K., Schuster, S. and Gilles, E. D. (2002) Metabolic network structure determines key aspects of functionality and regulation. Nature. 420, 190193

19 Trinh, C. T., Wlaschin, A. and Srienc, F. (2009) Elementary mode analysis: a useful metabolic pathway analysis tool for characterizing cellular metabolism. Appl Microbiol Biotechnol. 81, 813-826

20 Schuster, S., Hilgetag, C., Woods, J. H. and Fell, D. A. (2002) Reaction routes in biochemical reaction systems: algebraic properties, validated calculation procedure and example from nucleotide metabolism. J Math Biol. 45, 153-181 
21 Schwender, J., Goffman, F., Ohlrogge, J. B. and Shachar-Hill, Y. (2004) Rubisco without the Calvin cycle improves the carbon efficiency of developing green seeds. Nature. 432, 779-782

22 Teusink, B., Wiersma, A., Jacobs, L., Notebaart, R. A. and Smid, E. J. (2009) Understanding the adaptive growth strategy of Lactobacillus plantarum by in silico optimisation. PLoS Comput Biol. 5, e1000410

23 de Figueiredo, L. F., Schuster, S., Kaleta, C. and Fell, D. A. (2009) Can sugars be produced from fatty acids? A test case for pathway analysis tools. Bioinformatics. 25, 152158

24 Scheer, M., Grote, A., Chang, A., Schomburg, I., Munaretto, C., Rother, M., Söhngen, C., Stelzer, M., Thiele, J. and Schomburg, D. (2011) BRENDA, the enzyme information system in 2011. Nucleic Acids Res. 39, D670-676

25 Caspi, R., Altman, T., Dale, J. M., Dreher, K., Fulcher, C. A., Gilham, F., Kaipa, P., Karthikeyan, A. S., Kothari, A., Krummenacker, M., Latendresse, M., Mueller, L. A., Paley, S., Popescu, L., Pujar, A., Shearer, A. G., Zhang, P. and Karp, P. D. (2010) The MetaCyc database of metabolic pathways and enzymes and the BioCyc collection of pathway/genome databases. Nucleic Acids Res. 38, D473-479

26 van der Horst, A., Schavemaker, J. M., Pellis-van Berkel, W. and Burgering, B. M. (2007) The Caenorhabditis elegans nicotinamidase PNC-1 enhances survival. Mech Ageing Dev. 128, 346-349

27 Balan, V., Miller, G. S., Kaplun, L., Balan, K., Chong, Z. Z., Li, F., Kaplun, A., VanBerkum, M. F., Arking, R., Freeman, D. C., Maiese, K. and Tzivion, G. (2008) Life span extension and neuronal cell protection by Drosophila nicotinamidase. J Biol Chem. 283, 27810-27819

28 Bieganowski, P. and Brenner, C. (2003) The reported human NADsyn2 is ammoniadependent NAD synthetase from a pseudomonad. J Biol Chem. 278, 33056-33059

29 Revollo, J. R., Grimm, A. A. and Imai, S. (2007) The regulation of nicotinamide adenine dinucleotide biosynthesis by Nampt/PBEF/visfatin in mammals. Curr Opin Gastroenterol. 23, 164-170

30 Belenky, P., Bogan, K. L. and Brenner, C. (2007) NAD ${ }^{+}$metabolism in health and disease. Trends Biochem Sci. 32, 12-19

31 Belenky, P., Christensen, K. C., Gazzaniga, F., Pletnev, A. A. and Brenner, C. (2009) Nicotinamide riboside and nicotinic acid riboside salvage in fungi and mammals. Quantitative basis for Urh1 and purine nucleoside phosphorylase function in $\mathrm{NAD}^{+}$metabolism. J Biol Chem. 284, 158-164

32 Grubmeyer, C. T., Gross, J. W. and Rajavel, M. (1999) Energy coupling through molecular discrimination: nicotinate phosphoribosyltransferase. Methods Enzymol. 308, 2848

33 Burgos, E. S, and Schramm, V. L. (2008) Weak coupling of ATP hydrolysis to the chemical equilibrium of human nicotinamide phosphoribosyltransferase. Biochemistry. 47, 11086-11096

34 von Kamp, A. and Schuster, S. (2006) Metatool 5.0: fast and flexible elementary modes analysis. Bioinformatics. 22, 1930-1931

35 Tempel, W., Rabeh, W. M., Bogan, K. L., Belenky, P., Wojcik, M., Seidle, H. F., Nedyalkova, L., Yang, T., Sauve, A. A., Park, H. W. and Brenner, C. (2007) Nicotinamide riboside kinase structures reveal new pathways to $\mathrm{NAD}^{+}$. PLoS Biol. 5, e263

36 Ghislain, M., Talla, E. and François, J. M. (2002) Identification and functional analysis of the Saccharomyces cerevisiae nicotinamidase gene, PNC1. Yeast. 19, 215-224

37 Rongvaux, A., Shea, R. J., Mulks, M. H., Gigot, D., Urbain, J., Leo, O. and Andris, F. (2002) Pre-B-cell colony-enhancing factor, whose expression is up-regulated in activated 
lymphocytes, is a nicotinamide phosphoribosyltransferase, a cytosolic enzyme involved in NAD biosynthesis. Eur J Immunol. 32, 3225-3234

38 Revollo, J. R., Grimm, A. A. and Imai, S. (2004) The NAD biosynthesis pathway mediated by nicotinamide phosphoribosyltransferase regulates Sir2 activity in mammalian cells. J Biol Chem. 279, 50754-50763

39 Belenky, P., Racette, F. G., Bogan, K. L., McClure, J. M., Smith, J. S. and Brenner, C. (2007) Nicotinamide riboside promotes Sir2 silencing and extends lifespan via Nrk and Urh1/Pnp1/Meu1 pathways to NAD ${ }^{+}$. Cell. 129, 473-484

40 Berger, F., Lau, C., Dahlmann, M. and Ziegler, M. (2005) Subcellular compartmentation and differential catalytic properties of the three human nicotinamide mononucleotide adenylyltransferase isoforms. J Biol Chem. 280, 36334-36341 41 Bogan, K. L., Evans, C., Belenky, P., Song, P., Burant, C. F., Kennedy, R. and Brenner, C. (2009) Identification of Isn1 and Sdt1 as glucose- and vitamin-regulated nicotinamide mononucleotide and nicotinic acid mononucleotide 5'-nucleotidases responsible for production of nicotinamide riboside and nicotinic acid riboside. J Biol Chem. 284, 3486134869

42 Ramsey, K. M., Yoshino, J., Brace, C. S., Abrassart, D., Kobayashi, Y., Marcheva, B., Hong, H. K., Chong, J. L., Buhr, E. D., Lee, C., Takahashi, J. S., Imai, S. and Bass, J. (2009) Circadian clock feedback cycle through NAMPT-mediated NAD ${ }^{+}$biosynthesis. Science. 324, 651-654

43 Nakahata, Y., Sahar, S., Astarita, G., Kaluzova, M. and Sassone-Corsi, P. (2009) Circadian control of the NAD ${ }^{+}$salvage pathway by CLOCK-SIRT1. Science. 324, 654-657 44 Feist, A. M., Herrgård, M. J., Thiele, I, Reed, J. L. and Palsson, B. Ø. (2009) Reconstruction of biochemical networks in microorganisms. Nat Rev Microbiol. 7, 129-143 45 de Figueiredo, L. F., Podhorski, A., Rubio, A., Kaleta, C., Beasley, J. E., Schuster, S. and Planes, F. J. (2009) Computing the shortest elementary flux modes in genome-scale metabolic networks. Bioinformatics. 25, 3158-3165

46 Kaleta, C., de Figueiredo, L. F. and Schuster, S. (2009) Can the whole be less than the sum of its parts? Pathway analysis in genome-scale metabolic networks using elementary flux patterns. Genome Res. 19, 1872-1883

47 Faust, K., Croes, D. and van Helden, J. (2009) In response to 'Can sugars be produced from fatty acids? A test case for pathway analysis tools'. Bioinformatics. 25, 3202-3205

48 de Figueiredo, L. F., Schuster, S., Kaleta, C. and Fell, D. A. (2009) Response to comment on 'Can sugars be produced from fatty acids? A test case for pathway analysis tools'. Bioinformatics. 25, 3330-3331

49 Krehl, W. A., Teply, L. J. and Elvehjem, C. A. (1945) Corn as an Etiological Factor in the Production of a Nicotinic Acid Deficiency in the Rat. Science. 101, 283

50 Hara, N., Yamada, K., Shibata, T., Osago, H., Hashimoto, T. and Tsuchiya, M. (2007) Elevation of cellular NAD levels by nicotinic acid and involvement of nicotinic acid phosphoribosyltransferase in human cells. J Biol Chem. 282, 24574-24582

51 Nakagawa, T., Lomb, D. J., Haigis, M. C. and Guarente, L. (2009) SIRT5 deacetylates carbamoyl phosphate synthetase 1 and regulates the urea cycle. Cell. 137, 560-570

52 Evans, C., Bogan, K. L., Song, P., Burant, C. F., Kennedy, R. T. and Brenner, C. $\mathrm{NAD}+$ metabolite levels as a function of vitamins and calorie restriction: evidence for different mechanisms of longevity. BMC Chem Biol. 10, 2

53 Lu, S. P., Kato, M. and Lin, S. J. (2009) Assimilation of endogenous nicotinamide riboside is essential for calorie restriction-mediated life span extension in Saccharomyces cerevisiae. J Biol Chem. 284, 17110-17119 


\section{Tables and Figures}

\begin{tabular}{cccc}
\hline ATP & ADP & ribose-P & EFMs \\
\hline-2 & 1 & 0 & 21 \\
-4 & 3 & 0 & 22,29 \\
-4 & 3 & -1 & 19 \\
-5 & 4 & 0 & 30 \\
-5 & 4 & -1 & 26 \\
-6 & 5 & 0 & 23,27 \\
-6 & 5 & -1 & 20,25 \\
-7 & 6 & 0 & 24,28 \\
\hline
\end{tabular}

Table 1. Overall stoichiometry of ATP and ribose phosphate in AAD $^{+}$synthesis pathways. Negative and positive values correspond to consumption and production, respectively. AMP production as well as the production of ribose were converted to extra dephosphorylation of ATP. 


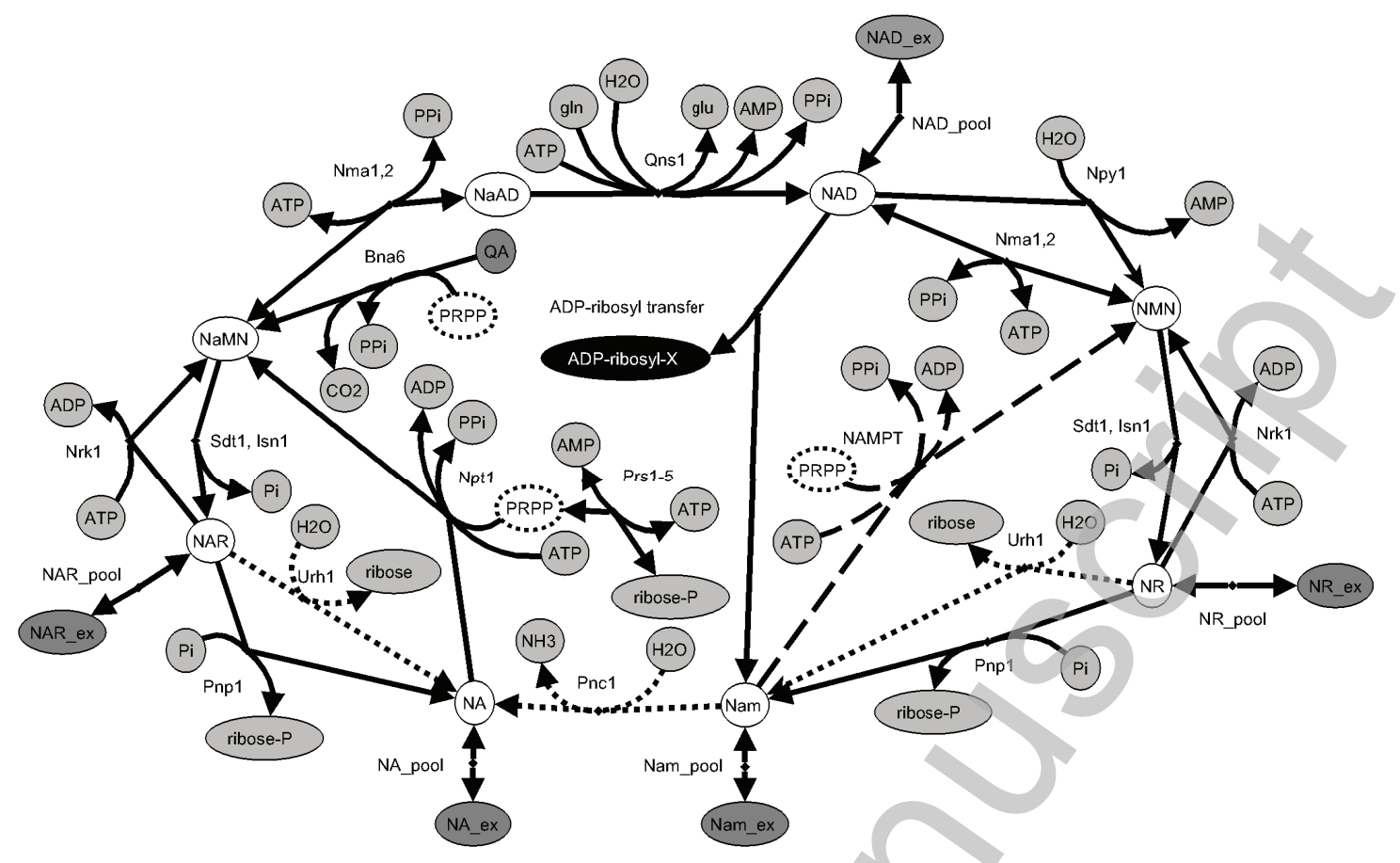

Fig.1. Overview of $\mathrm{NAD}^{+}$metabolism as considered here for modelling. See List of Abbreviations for names of metabolites and enzymes. Irreversible and reversible reactions are indicated by unidirectional and bidirectional arrows, respectively. White ellipses, internal metabolites; black ellipse, ADP-ribosyl-X designates the formation of ADP-ribose derivatives from $\mathrm{NAD}^{+}$in various signalling reactions; dark grey ellipses, external metabolites representing the pool of $\mathrm{NAD}^{+}$and its precursors; light grey ellipses, other external metabolites. Gene name abbreviations correspond to yeast except for the reaction catalyzed by nicotinamide phosphoribosyltransferase (NAMPT) which is present in human, but not yeast. Dashed arrow, reaction specific to humans; dotted arrows, reactions specific to yeast. The graph was drawn with yEd software package. 
a)

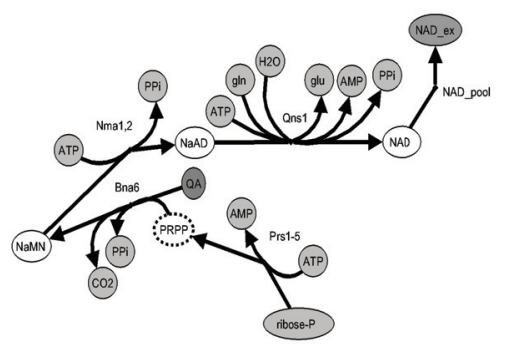

c)

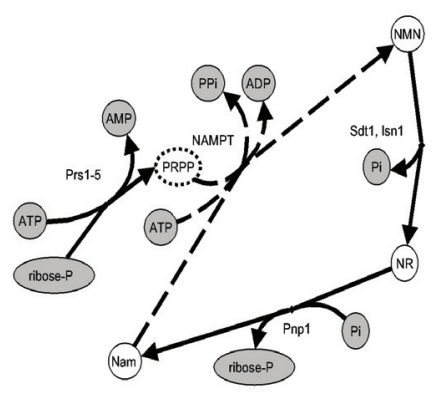

e)

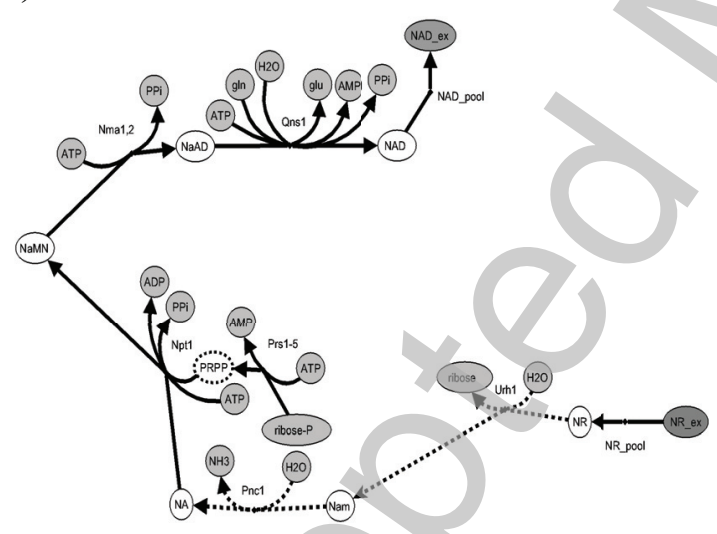

g)

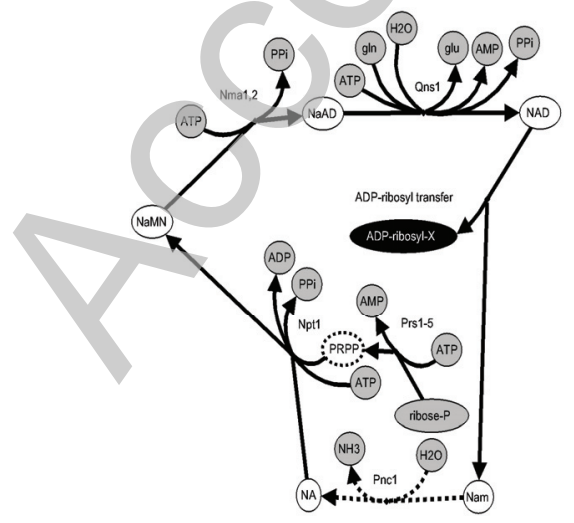

b)

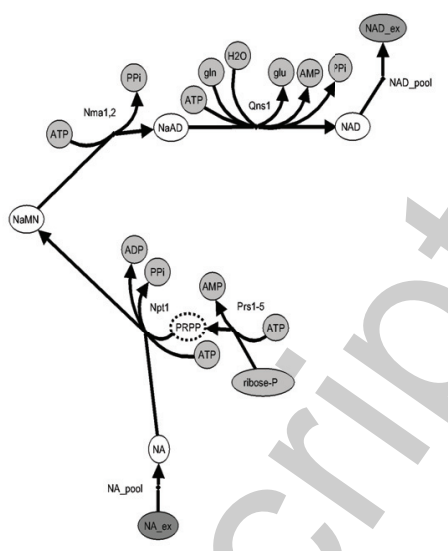

d)

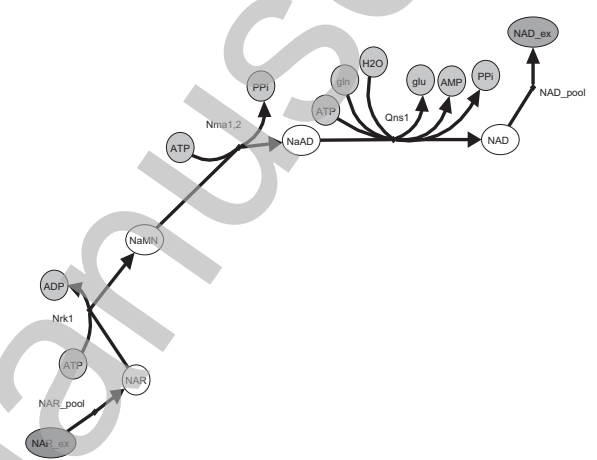

f)

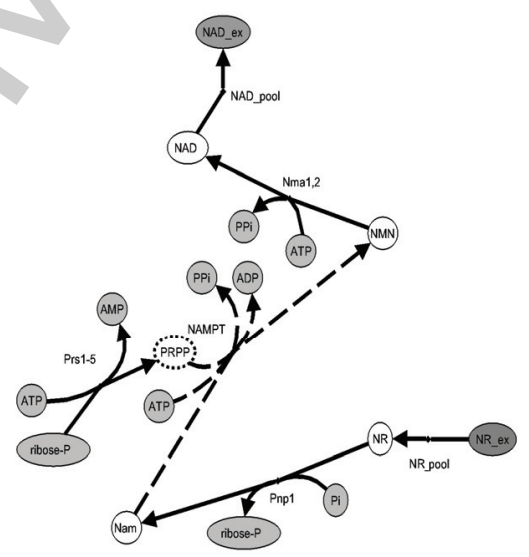

h)

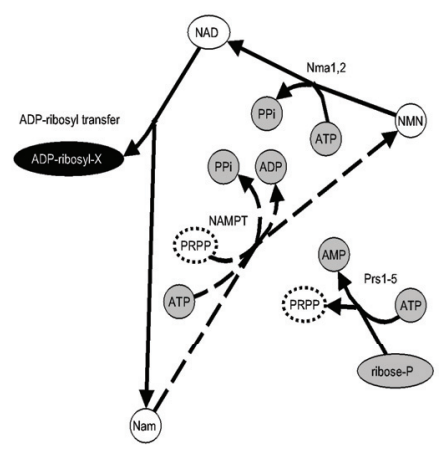

Fig. 2. Visualization of elementary flux modes in $\mathrm{NAD}^{+}$metabolism. (a) de novo biosynthesis of $\mathrm{NAD}^{+}$from $\mathrm{QA}$, which is generated from tryptophan in the kynurenine 
pathway, mode 26; (b) Preiss-Handler pathway, mode 25; (c) futile cycle in human, mode 47; (d) Nrk-dependent pathway for NAR salvage (Tempel et al. 2007), mode 22; (e) Nrkindependent $\mathrm{NAD}^{+}$pathway of NR utilization in yeast (Belenky et al. 2007b), mode 28; f) Nrk-independent $\mathrm{NAD}^{+}$salvage pathway in humans, mode 29; (g) steady-state signalling pathway in yeast, mode 51; (h) steady-state signalling pathway in human, mode 52 . Dashed arrow, reaction specific to humans; dotted arrows, reactions specific to yeast. 

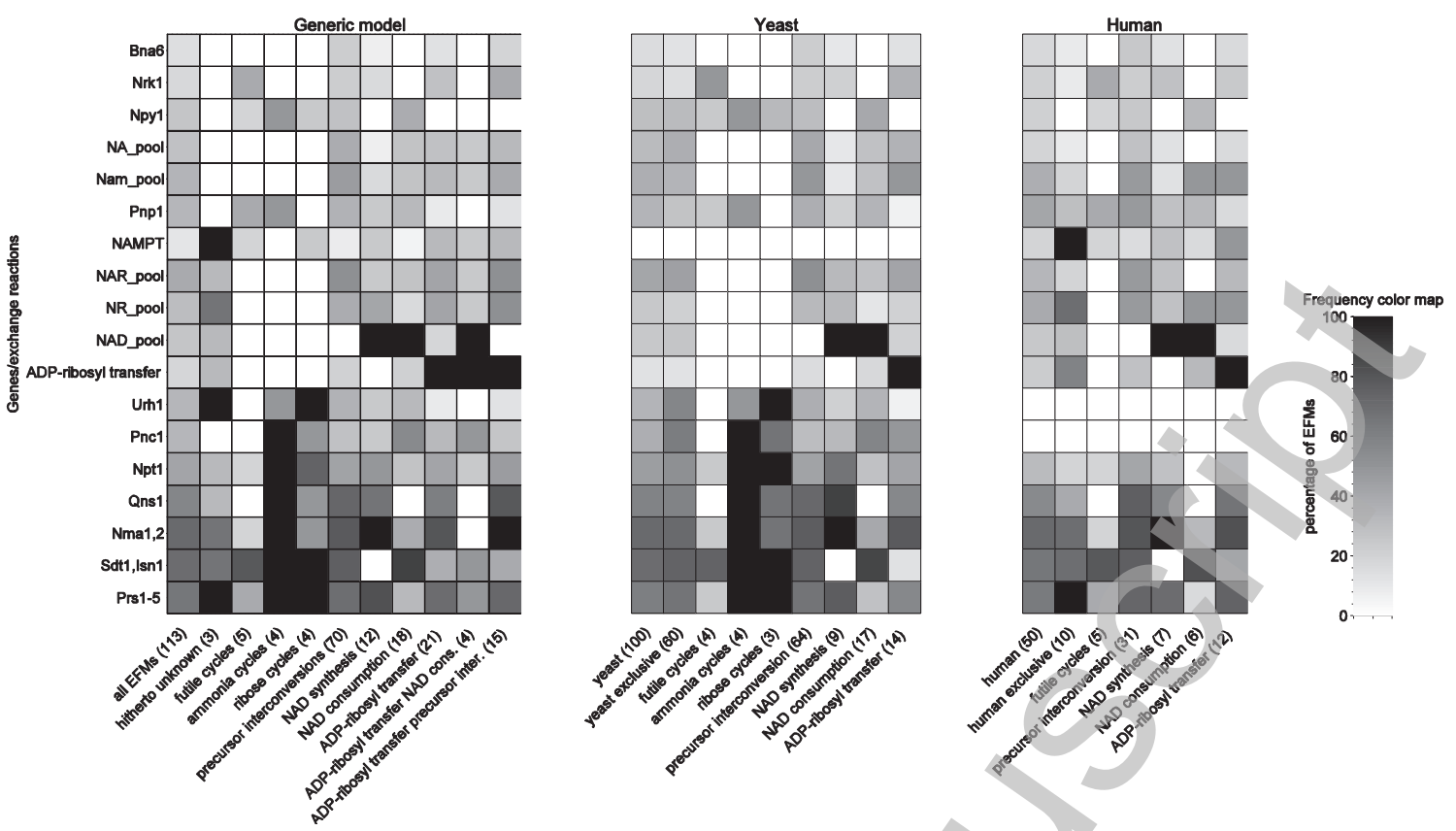

Fig. 3. Presence of enzymes/exchange reactions in each EFM subset. The numbers in brackets correspond to the number of EFMs present in the subset. Only futile cycles in the "strict sense" (see text) are shown here. Futile cycles in the broader sense are here classified amidating/deamidating cycles (termed "ammonia cycles") and cyclic pathways consuming ribose-P and releasing ribose (termed "ribose cycles"). All the pathways termed "ADP-ribosyl transfer" involve the reaction with the same name. The pathways additionally specified as "NAD cons." or "precursor inter." involve NAD consumption or precursor interconversion, respectively. The colour code continuously ranges from black (enzyme is absent from all EFMs) to white (present in all EFMs). 